\title{
The OU-ISIR Gait Database Comprising the Large Population Dataset and Performance Evaluation of Gait Recognition
}

\author{
Haruyuki Iwama, Mayu Okumura, Yasushi Makihara, and Yasushi Yagi, Member, IEEE
}

\begin{abstract}
This paper describes the world's largest gait database - the "OU-ISIR Gait Database, Large Population Dataset" - and its application to a statistically reliable performance evaluation of vision-based gait recognition. Whereas existing gait databases include at most 185 subjects, we construct a larger gait database that includes 4007 subjects (2135 males and 1872 females) with ages ranging from 1 to 94 years. The dataset allows us to determine statistically significant performance differences between currently proposed gait features. In addition, the dependences of gait-recognition performance on gender and age group are investigated and the results provide several novel insights, such as the gradual change in recognition performance with human growth.
\end{abstract}

Index Terms-Gait database, gait recognition, large population, performance evaluation.

\section{INTRODUCTION}

B IOMETRIC-BASED person-recognition techniques have become increasingly important in crime prevention and investigation. Gait, as a biometric cue, has relatively recently attracted much attention and it is expected to be applied to widearea surveillance and crime investigation owing to the possibility of identifying subjects from a distance without the cooperation of the subjects. Thus, vision-based gait-recognition approaches have been widely developed in recent years [1]-[9].

For the development and statistically reliable evaluation of gait-recognition approaches, the construction of a common gait database is essential. There are two considerations in constructing a gait database: (1) the variation in walking conditions (e.g., view, speed, clothing, and carrying conditions), and (2) the number and diversity of the subjects. The first

Manuscript received December 12, 2011; revised May 02, 2012; accepted May 14, 2012. Date of publication June 11, 2012; date of current version September 07, 2012. This work was supported in part by Grant-in-Aid for Scientific Research (S) 21220003 and the "R\&D Program for Implementation of Anti-Crime and Anti-Terrorism Technologies for a Safe and Secure Society," Strategic Funds for the Promotion of Science and Technology of the Ministry of Education, Culture, Sports, Science and Technology, the Japanese Government. The associate editor coordinating the review of this manuscript and approving it for publication was Dr. Fabio Scotti.

The authors are with the Department of Intelligent Media, Institute of Scientific and Industrial Research, Osaka University, Ibaraki, Osaka, 567-0047, Japan (e-mail: iwama@am.sanken.osaka-u.ac.jp; okumura@am.sanken.osaka-u.ac.jp; yagi@am.sanken.osaka-u.ac.jp)

Color versions of one or more of the figures in this paper are available online at http://ieeexplore.ieee.org.

Digital Object Identifier 10.1109/TIFS.2012.2204253 consideration is important in evaluating the robustness of the gait recognition, because walking conditions depend on the time and circumstances and often differ between gallery and probe. For instance, the clothing and carrying conditions when walking along a street in a suit with a bag while on business can differ from those when strolling empty-handed in casual clothes during leisure time. The second consideration is important to ensure statistical reliability of the performance evaluation. Moreover, if the database is used for soft biometric applications such as gait-based gender and age classification [10], [11], the diversity of subjects in terms of gender and age plays a significant role in the performance evaluation.

Although several gait databases have been constructed [12]-[25], with most of these taking good account of the first consideration, the second consideration is still insufficiently addressed since these databases include at most 185 subjects [24] and the subjects' genders and ages are biased in many of the databases. The exceptions are the large-scale datasets introduced in [26] and [27], which do address the second consideration and include respectively, 1,035 and 1,728 subjects with ages ranging from 2 to 94 years. In these datasets, however, the gait images are captured using cameras with varying poses (e.g., a camera's pose on one day differs slightly from that on another day, or some subjects are captured using first one camera and then another with a slightly different pose) and this could introduce bias into the evaluation results.

In this paper, we focus on the second consideration and introduce a large population dataset that is a major upgrade to previously reported large-scale datasets in [26] and [27]. The extensions of this dataset are as follows.

1) The number of subjects is considerably greater in the dataset; i.e., there are more than thrice the number of subjects in the dataset in [26] and more than twice the number in the dataset in [27].

2) All silhouette images are normalized with respect to the image plane to remove the bias of camera rotation for more equitable performance evaluation.

3) The observation angle of subjects in each frame is specifically defined for the sake of fair analysis in terms of the observation angle, whereas previous works merely defined the angle as a side view.

Our dataset is the largest gait dataset in the world, comprising over 4,000 subjects of both genders and including a wide range of ages. Although the dataset does not include any variations in walking conditions, it allows us to investigate the upper limit of gait-recognition performance in a more statistically reliable way 
TABLE I

EXISTING MAJOR GAIT DATABASES

\begin{tabular}{l|r|l}
\hline Database & \#Subjects & Data covariates \\
\hline \hline Soton database & $12[15]$ & 4 views, 5 shoes, 3 clothes, 5 bags (including w/o), 3 speeds \\
& $115[14]$ & 3 scenario (outdoor, indoor track, treadmill), 2 views per scenario \\
& $25[28]$ & Time (0, 1, 3, 4, 5, 8, 9, and 12 months), 12 views \\
\hline USF dataset & $122[18]$ & 2 views, 2 shoes, 2 surfaces, baggage (w/ and w/o), time (6 months) \\
\hline CASIA dataset & $20[16]$ & 3 views \\
& $124[19]$ & 11 views, clothing (w/ and w/o coat), baggage (w/ and w/o) \\
& $153[20]$ & 3 speeds, baggage (w/ and w/o), \\
\hline OU-ISIR Gait Database, & $34[22]$ & 9 speeds (2, 3, 4, 5, 6, 7, 8, 9, and 10 km/h) \\
Treadmill dataset & $68[23]$ & 32 clothes combination at most \\
& $185[24]$ & Gait fluctuation among periods \\
& $168[25]$ & 25 views \\
\hline
\end{tabular}

and to reveal how gait-recognition performance differs between genders and age groups. Thus, our dataset can contribute much to the development of gait-based applications, and we demonstrate its validity through experiments with state-of-the-art gait representations.

The outline of the paper is as follows. Section II introduces existing gait databases, while Section III addresses the construction of the dataset. The gait-recognition approach for performance evaluation is described in Section IV, and various performance evaluations using our dataset are presented in Section V. Section VI presents our conclusions and discusses future work.

\section{RELATED WORK}

Existing major gait databases are summarized in Table I. Here, we briefly describe these databases.

The Soton database is composed of a small population dataset [15] and a large population dataset [14]. The small dataset contains subjects walking around an indoor track, with each subject filmed wearing a variety of footwear and clothing, carrying various bags, and walking at different speeds. Hence, the database is used for exploratory factor analysis of gait recognition [29]. The large dataset was the first gait database to contain over 100 subjects and has contributed to the study of gait recognition mainly in terms of intersubject variation. The recently published Soton Temporal database [21] contains the largest time variations; up to 12 months to date [28]. It enables the investigation of the effect of time on the performance of gait biometrics, allowing the use of 3-D volumetric data.

The USF dataset [18] is one of the most widely used gait datasets and is composed of a gallery and 12 probe sequences captured outdoors under different walking conditions including factors such as view, shoes, surface, baggage, and time. As the number of factors is the largest of all existing databases, despite there being only two variations for each factor, the USF database is suitable for the evaluation of the interfactor effect, as opposed to the intrafactor effect, on gait-recognition performance.

The CASIA database, Dataset A [16] contains image sequences from three views and can be used for the analysis of the effect of the view angle on recognition performance. The CASIA database, Dataset B [19] consists of multiview (11 views) walking sequences and includes variations in the view angle, clothing, and carrying conditions. Since it contains the finest azimuth view variations, it is useful for the analysis and modeling of the effect of view on gait recognition [30]. The CASIA database, Dataset C [20] was the first database to include infrared gait images captured at night, thus enabling the study of night gait recognition.

The OU-ISIR Gait Database, Treadmill Dataset [22]-[25] contains gait images of subjects on a treadmill with the largest range of view variations (25 views: 12 azimuth views times 2 tilt angles, plus 1 top view), speed variations (9 speeds: $1 \mathrm{~km} / \mathrm{h}$ intervals between 2 and $10 \mathrm{~km} / \mathrm{h}$ ), and clothing variations (up to 32 combinations), and as such, it can be used to evaluate view-invariant [4], speed-invariant [22] and clothing-invariant [23] gait recognition. In addition, it is used to analyze gait features in gender and/or age-group classification [25], since the diversities of gender and age of the subjects are greater than those in currently available gait databases.

Next, we review the number and diversity of subjects. Table I shows that existing major databases include more than 100 subjects. Although these databases are statistically reliable to some extent, the number of subjects is insufficient when compared with databases of other biometrics such as fingerprints and faces. In addition, the populations of genders and ages are biased in many of these databases; e.g., there are no children in the USF dataset with most of the subjects in their twenties and thirties, while the ratio of males to females is 3 to 1 in the CASIA dataset (Dataset B). Such biases are undesirable in performance evaluation of gait-based gender and age-group estimation and in performance comparison of gait recognition between genders and age groups.

\section{The OU-ISIR Gait Database, LARge Population DATASET}

\section{A. Capture System}

An overview of our capture system is illustrated in Fig. 1. Each subject was asked to walk at his or her own preferred speed through a straight course (red arrows) at most twice under the same conditions. The length of the course was approximately $10 \mathrm{~m}$, with approximately $3 \mathrm{~m}$ (at least $2 \mathrm{~m}$ ) sections at the beginning and end regarded as acceleration and deceleration 


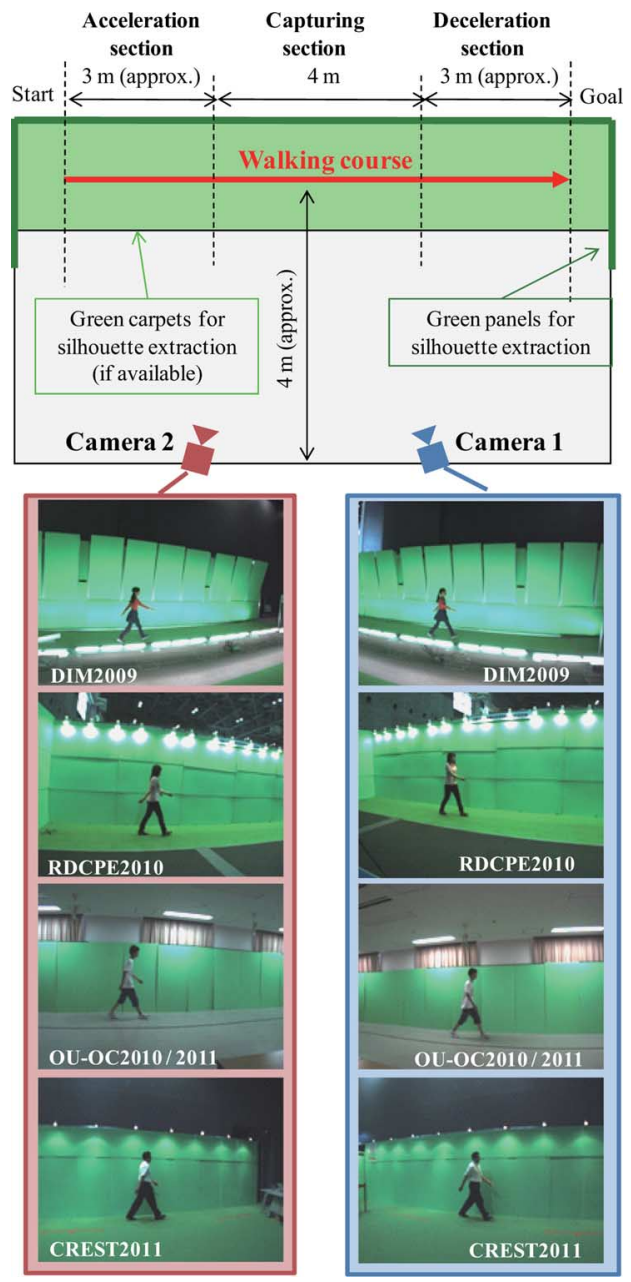

Fig. 1. Overview of capture system and captured images.

zones, respectively. Two cameras were set approximately $4 \mathrm{~m}$ from the walking course to observe (1) the transition from a front-oblique view to a side view (camera 1), and (2) the transition from a side view to a rear-oblique view (camera 2). We used Flea2 cameras manufactured by Point Gray Research Inc. with HF3.5M-2 lenses manufactured by SPACE Inc. The image size and frame rate were, respectively, $640 \times 480$ pixels and $30 \mathrm{fps}$. The recorded image format was uncompressed bitmap. Moreover, green background panels and carpet (if available) were arranged along the walking course for the purpose of clear silhouette extraction.

\section{B. Data Collection}

The dataset was collected during entertainment-oriented demonstrations of an online gait personality measurement [31] at outreach activity events in Japan, including the Dive Into the Movie project (DIM2009) [32], the 5th Regional Disaster and Crime Prevention Expo (RDCPE2010), Open Campus at Osaka University (OU-OC2010/2011), and the Core Research for Evolutional Science and Technology project (http://www.jst.go.jp/kisoken/crest/en/index.html, CREST2011). All the events were held at indoor halls and the numbers of visitors at each event are summarized in Table II.

Each subject was requested to give their informed consent permitting the use of the collected data for research purposes.
Also, the age and gender of each subject were collected as metadata. All the subjects walked empty-handed, wearing their own clothing (some subjects wore a hat) and footwear. Examples of images captured at each event are shown in Fig. 1.

\section{Statistics}

From the data collected by camera 1 (images were taken with two cameras at the events), the world's largest gait dataset of 4,007 subjects (2,135 males and 1,872 females) with ages ranging from 1 to 94 years was constructed. We call this dataset the "OU-ISIR Gait Database, Large Population Dataset C1 Version I"1, which we abbreviate to OULP-C1V12. Detailed distributions of the subjects' gender and age are shown in Fig. 2, while example images of the subjects are shown in Fig. 3. Almost all the subjects are of Asian descent.

The dataset comprises two subsets, which we call OULP-C1V1-A and OULP-C1V1-B. OULP-C1V1-A is a set of two sequences (gallery and probe sequences) per subject and is intended for use in evaluating gait-recognition performance under almost constant walking conditions. OULP-C1V1-B is a set of one sequence per subject and is intended for use in investigating gait-based gender classification and age estimation. OULP-C1V1-A and OULP-C1V1-B are major upgrades to the datasets introduced in [26] and [27], respectively. For brevity, we omit the description of the dataset header "OULP-C1V1-".

Each of the main subsets is further divided into five subsets based on the observation angle (55 [deg], 65 [deg], 75 [deg], 85 [deg], and including all four angles) of each subject. We call these subsets $\mathbf{A} / \mathbf{B}-\mathbf{5 5}, \mathbf{A} / \mathbf{B}-\mathbf{6 5}, \mathbf{A} / \mathbf{B}-\mathbf{7 5}, \mathbf{A} / \mathbf{B}-\mathbf{8 5}$, and $\mathbf{A} / \mathbf{B}-\mathbf{A L L}$, respectively, with each subject belonging to at least one of these subsets. The observation angle $\theta_{s}$ of each subject in each frame is defined by the y-axis of the world coordinate system (which is parallel to the walking direction) and the line of sight of the camera as illustrated in Fig. 4.

A subject is included in a bin of a subset if one gait period occurs in the range of angles (as illustrated in Fig. 4) corresponding to that subset. For example, if a subject is recorded twice (both gallery and probe sequences) with a complete gait period in the range of 55 [deg], the subject is included in a bin of $\mathbf{A - 5 5}$ and one of B-55. Moreover, if a subject is recorded twice with a complete gait period covering all the angle ranges, the subject is included in a bin of all the subsets. A gait period is calculated from the whole sequence (see Section IV-B for details on the calculation of the gait period).

An example image for each observation angle is shown in Fig. 4, while a breakdown of the number of subjects is given in Table III. In this table, the values in the "Total" column represent the number of subjects included in at least one of the subsets of 55 [deg], 65 [deg], 75 [deg], and 85 [deg]. As mentioned above, the numbers of subjects for dataset $\mathbf{A}$ represent those that have been recorded twice. Also, the differences between datasets $\mathbf{A}$ and $\mathbf{B}$ for each subset represent the numbers of subjects recorded

\footnotetext{
${ }^{1}$ To be prepared for publication. The data will be published in the form of normalized silhouette image sequences in PNG format, with a total data size of about $1.5 \mathrm{~GB}$.

${ }^{2}$ The naming format is OULP-[camera ID][version ID]-[headerl][header2]-...
} 
TABLE II

VISITORS AT EVENTS

\begin{tabular}{llr}
\hline Event & Term & \#Visitors (approx.) \\
\hline \hline DIM2009 & 3 days in March 2009 & 1,600 \\
RDCPE2010 & 2 days in June 2010 & 280 \\
OU-OC2010 & 1 day in August 2010 & 70 \\
OU-OC2011 & 1 day in August 2011 & 90 \\
CREST2011 & 5 days in August 2011 & 2,000 \\
\hline
\end{tabular}

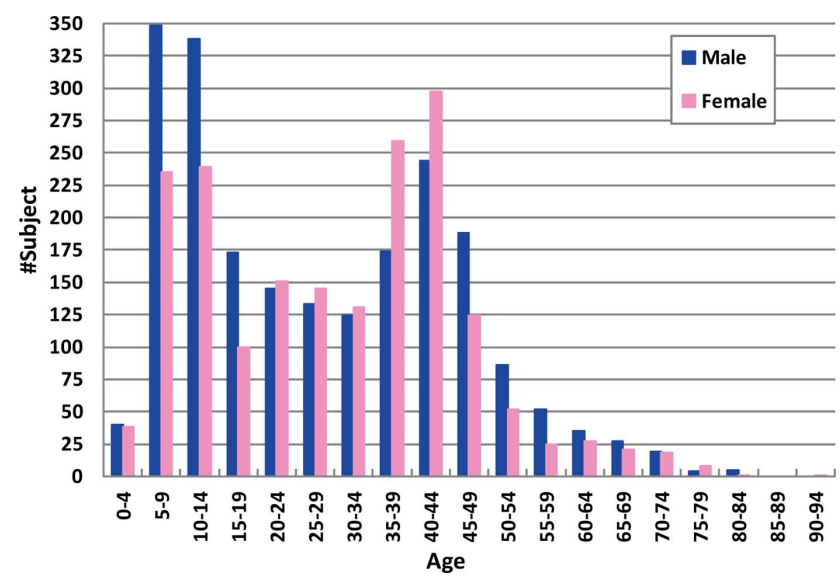

Fig. 2. Distributions of the subjects' gender and age in OULP-C1V1.

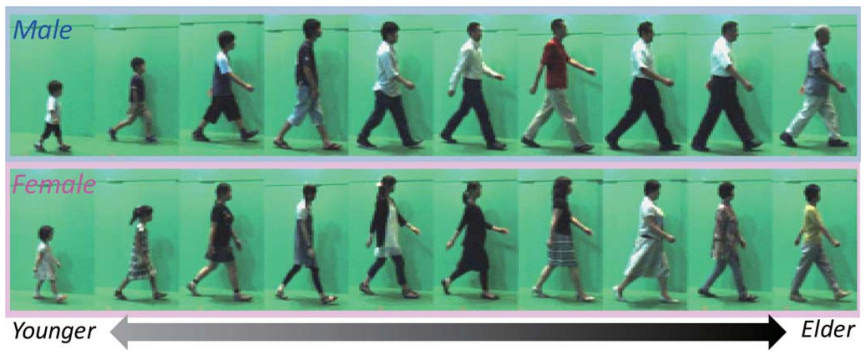

Fig. 3. Examples of subjects in OULP-C1V1.

only once. Take for example, the subset of 55 [deg] in Table III (A-55 and B-55) where 3,706 subjects are recorded twice and 292 subjects are recorded only once. Note that there are also differences in the numbers of subjects between subsets, because the sequence length and observation angles for each subject are not exactly the same.

\section{Advantages}

Compared with existing gait databases, our dataset has the following strengths.

1) Large population: The number of subjects is more than 20 times that in publicly available large-scale gait databases. This improves the statistical reliability of various performance evaluations such as the comparison of gait recognition.

2) Gender balance: The ratio of males to females is close to 1 . This is a desirable property for more reliable performance evaluation of gait-based gender classification and for comparison of gait-recognition performance between genders.

3) Whole generation: The age range is from 1 to 94 years with each 10-year interval up to 49 years of age containing more than 400 subjects (even in the smallest

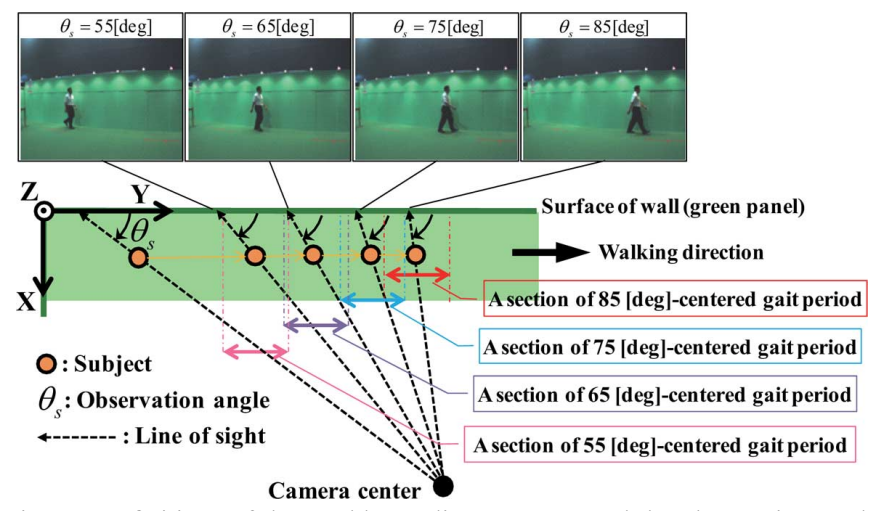

Fig. 4. Definitions of the world coordinate system and the observation angle of a subject, and an example image at each observation angle. The $Y-Z$ plane corresponds to the background wall behind the walking subjects, while the $X-Y$ plane corresponds to the ground plane.

subset A-ALL). In addition, it is noteworthy that our dataset includes a sufficient number of children at all stages of growth, whereas other large-scale gait databases are mainly composed of adult subjects. This provides more statistically reliable results for gait-based age-group classification and comparisons of the difficulties in gait recognition among age groups.

4) Silhouette quality: The quality of each silhouette image is relatively high because we visually checked each silhouette more than twice and made manual modifications if necessary. This enables the elimination of silhouette quality problems from gait analysis. On the contrary, the silhouette images in most of the existing public databases are automatically extracted and often include significant over/under-segmentation. Although manually modified silhouettes were created in the investigation of the effect of silhouette quality on gait recognition in [33] and [34], these have not been published.

\section{E. Preprocessing}

This section briefly describes the method used for size-normalized silhouette extraction.

1) Silhouette Extraction: The first step involved extraction of gait silhouette images via graph-cut-based segmentation [35] in conjunction with background subtraction. Of course, over/ under-segmentation errors appeared in some extracted silhouette images. Hence, as described above, we visually checked all silhouette images at least twice and then manually modified under/over-segmentation if necessary. In more detail, a silhouette was shown to the observer in the form of a composite image in which the silhouette contour was overlaid on the corresponding original image. The observer checked whether the silhouette contour fitted the visually perceived human contour and if not, modified it using a GUI tool specially developed for this purpose.

2) Correction of Camera Rotation: In the second step, image normalization, including the correction of distortion and camera rotation, was carried out. Because the camera pose in the world coordinate system for each day/event was not strictly the same, we normalized the camera rotations in all silhouette images such that the image plane in each is parallel with the $Y-Z$ plane in the world coordinate system as shown in Fig. 5. 
TABLE III

BREakdown of the Number of SubJects in OULP-C1V1 (Male/Female)

\begin{tabular}{l|ccccc|c}
\hline \multirow{2}{*}{ Dataset } & \multicolumn{5}{|c|}{ Observation angle } & Tll \\
\hline \hline LP-C1V1-A & $55[\mathrm{deg}]$ & $65[\mathrm{deg}]$ & $75[\mathrm{deg}]$ & $85[\mathrm{deg}]$ & Total \\
& 3,706 & 3,770 & 3,751 & 3,249 & 3,141 & $\mathbf{3 , 8 3 5}$ \\
& $(1,977 / 1,729)$ & $(2,007 / 1,763)$ & $(1,995 / 1,756)$ & $(1,688 / 1,561)$ & $(1,644 / 1,497)$ & $(2,032 / 1,803)$ \\
LP-C1V1-B & 3,998 & 4,005 & 4,002 & 3,923 & 3,904 & $\mathbf{4 , 0 0 7}$ \\
& $(2,129 / 1,869)$ & $(2,133 / 1,872)$ & $(2,133 / 1,869)$ & $(2,073 / 1,850)$ & $(2,061 / 1,843)$ & $(2,135 / 1,872)$ \\
\hline
\end{tabular}

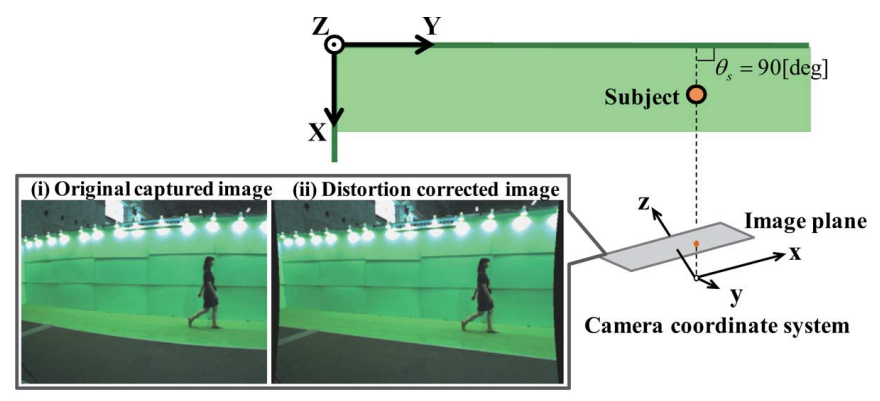

(a)

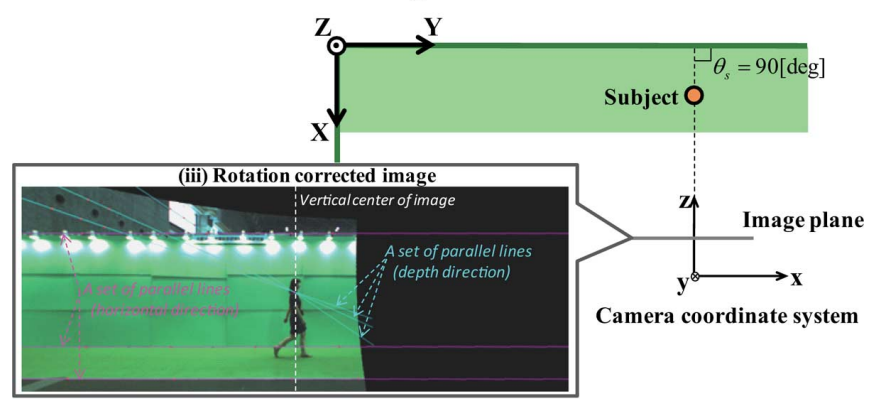

(b)

Fig. 5. Examples of the original and normalized camera pose, image plane, and images. In the rotation-corrected image in (b), the set of cyan lines and set of magenta lines represent the sets of parallel lines in the scene used to determine the vanishing points, while the white dashed line represents the vertical center line of the image. The observation angle is 90 [deg] at this line. (a) Original image plane. (b) Normalized image plane.

First, the intrinsic parameters of the camera and coefficients of lens distortion were estimated [36 $]^{3}$ and distortion corrected. An example of an undistorted image is shown in Fig. 5(a). The transformation matrix of camera rotation from the original pose (shown in Fig. 5(a)) to the target pose (shown in Fig. 5(b)) was then estimated for each day/event from the undistorted image using a pair of vanishing points [37] (i.e., horizontal and vertical vanishing points), estimated from the sets of parallel lines in the scene [38]. Finally, all the image pixels in the original image plane were reprojected onto the normalized image plane. An example of a camera rotation corrected image is shown in Fig. 5(b). Also, examples of a subject in each dataset after rotation correction are shown in Fig. 6.

3) Registration and Size Normalization: The third step involved registration and size normalization of the silhouette images [4]. First, the top, bottom, and horizontal center of the silhouette regions were obtained for each frame. The horizontal

\footnotetext{
${ }^{3}$ Calibration procedures were implemented using OpenCV version 1.1 functions.
}

center was chosen as the median of the horizontal positions belonging to the region. Second, a moving-average filter was applied to these positions. Third, we normalized the size of the silhouette images such that the height was just 128 pixels according to the average positions, and the aspect ratio of each region was maintained. Finally, we produced an $88 \times 128$ pixel image in which the average horizontal median corresponds to the horizontal center of the image. Examples of size-normalized silhouettes are shown in Fig. 7.

\section{GAIT RECOGNITION}

This section describes a framework for performance evaluation of gait recognition.

\section{A. Gait Features}

The current trend in gait representation is appearance and period-based representation, such as the averaged silhouette [39], also known as the Gait Energy Image (GEI) [40]. In this paper, we deal with six such state-of-the-art gait features: GEI, Frequency-Domain Feature [4] (referred to as FDF in this paper), Gait Entropy Image (GEnI) [5], Masked GEI based on GEnI [7] (referred to as MGEI in this paper), Chrono-Gait Image (CGI) [6], and Gait Flow Image (GFI) [8].

The GEI is obtained by averaging silhouettes over a gait cycle, while the FDF is generated by applying a Discrete Fourier Transform of the temporal axis to the silhouette images in a gait cycle. In this paper, 0,1 , and 2 times frequency elements are used. The GEnI is computed by calculating Shannon entropy for every pixel over a gait cycle, where the value of the GEI is regarded as the probability that the pixel takes the binary value. The MGEI is computed by masking the GEI with a pair-wise mask generated by each pair of probe and gallery GEnIs. The GEnI and MGEI aim to select the dynamic area from the GEI. The CGI is a temporal template in which the temporal information among gait frames is encoded by a color mapping function, and is obtained by compositing the color encoded gait contour images in a gait cycle. The GFI is based on an optical flow field from silhouettes representing motion information and is created by averaging the binarized flow images over a gait cycle. An example of each feature is shown in Fig. 8.

\section{B. Gait Period Detection}

For the quantification of periodic gait motion, we adopted the Normalized Auto Correlation (NAC) of the size-normalized 


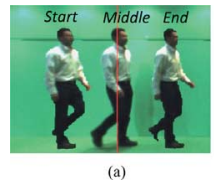

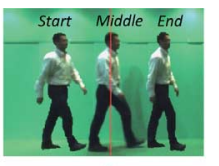

(b)

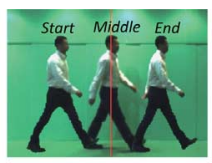

(c)

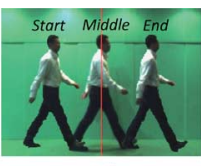

(d)
Fig. 6. Composite images showing examples of a subject in each dataset after rotation correction. Each composite image includes the subject at the start ("Start"), the middle ("Middle"), and the end ("End") of the section. The vertical red line represents the center of the section. (a) A-55. (b) A-65. (c) A-75. (d) A-85.

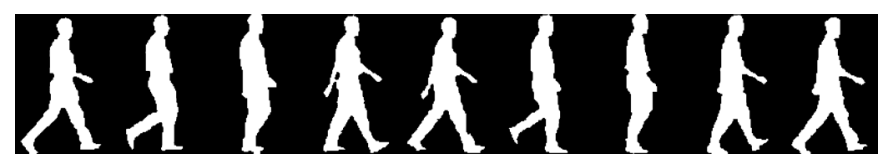

Fig. 7. Examples of size-normalized gait silhouettes (every four frames).

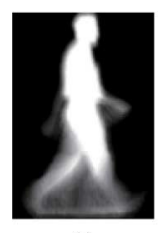

(a)

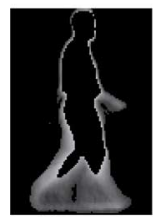

(d)

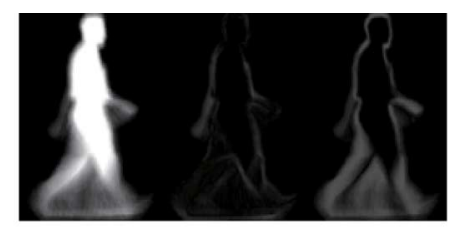

(b)

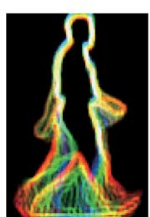

(e)

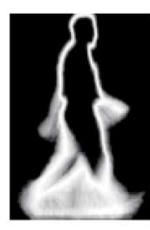

(c)
Fig. 8. Examples of gait features. (a) GEI. (b) FDF (three frequencies) (c) GEnI. (d) MGEI. (e) CGI. (f) GFI.

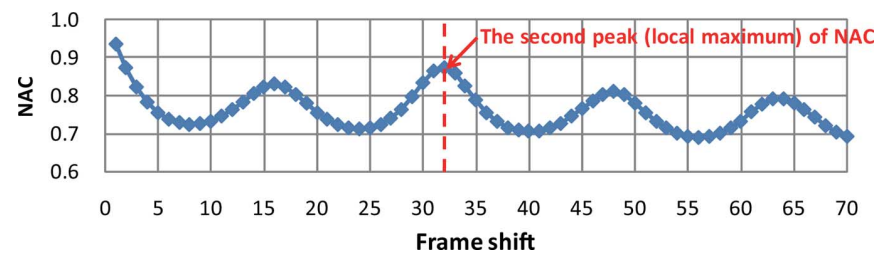

Fig. 9. Example of the relation between NAC and frame shift. In this figure, the frame shift corresponding to the second peak of the NAC is 32 .

silhouette images for the temporal axis:

$$
C(N)=\frac{\sum_{x, y} \sum_{n=0}^{T(N)} g(x, y, n) g(x, y, n+N)}{\sqrt{\sum_{x, y}^{T(N)} g(x, y, n)^{2}} \sqrt{\sum_{x=0} \sum_{n=0}^{T(N)} g(x, y, n+N)^{2}}}
$$

$$
T(N)=N_{\text {total }}-N-1
$$

where $g(x, y, n)$ is the silhouette value at position $(x, y)$ of the $n$-th frame, $C(N)$ is the autocorrelation for the $N$-frame shift, and $N_{\text {total }}$ is the total number of frames in the sequence. Because gait is a symmetrical motion to some extent, peaks of the NAC were assumed to appear for all half periods on the temporal axis. Thus, we determined the gait period $N_{\text {gait }}$ as the frame shift corresponding to the second peak of the NAC. An example of the relation between NAC and frame shift is shown in Fig. 9.

\section{Distance Matching}

In the evaluation of datasets A-55, A-65, A-75, and $\mathbf{A - 8 5}$, a gait feature for a subject was created from a section of a dataset (as illustrated in Fig. 6) that includes one gait period. Note that there is some area of overlap for some subjects between sections as shown in Fig. 4. All pairs of features (gallery and probe features) were then directly matched ${ }^{5}$.

The distance $D_{i j, K}$ between the $i$-th probe subject and the $j$-th gallery subject in dataset $K \quad \in \quad\{\mathbf{A}-\mathbf{5 5}, \mathbf{A - 5 5}, \mathbf{A}-\mathbf{6 5}, \mathbf{A}-\mathbf{7 5}, \mathbf{A}-85\}$ was measured as,

$$
D_{i j, K}=\left\|\mathbf{P}_{i, K}-\mathbf{G}_{j, K}\right\|_{2},
$$

where $\mathbf{P}_{i, K}$ and $\mathbf{G}_{j, K}$ are feature vectors of the $i$-th probe and $j$-th gallery in dataset $K$, respectively, and $\|\cdot\|_{2}$ is the Euclidean distance. In addition, we exploited z-normalization [41] of the distance among galleries for each probe to improve the performance in a one-to-one matching scenario.

For dataset A-ALL, we first calculated z-normalized distances for each section of the four above-mentioned datasets and then averaged them as a total distance. Note that this averaging is equivalent to combining the normalized scores via the sum rule [42].

\section{Performance Evaluation of Gait Recognition}

Despite the recent welcome development in gait recognition in the research community, the following open issues still remain.

1) An evaluation of gait recognition with statistical reliability has not been carried out owing to the lack of a large population dataset.

2) Also, to the best of our knowledge, the effects of gender and age on recognition performance have not been explored because of the lack of a dataset with sufficient subject diversity.

Therefore, we address the above issues using our dataset. In this section, we first show the statistical reliability of the evaluation using our database. The upper limits of recognition performance of state-of-the-art gait representations introduced in the previous section are then demonstrated. Finally, we reveal the effects of age and gender on recognition performance.

\section{A. Effect of the Number of Subjects}

First, the effect of the number of subjects is demonstrated by means of a Receiver Operating Characteristic (ROC) curve. The ROC curve is a common tool for performance evaluation in biometrics and denotes the trade-off between the False Rejection Rate (FRR) and False Acceptance Rate (FAR) when the acceptance threshold is changed by a receiver in a one-to-one matching scenario.

\footnotetext{
${ }^{4}$ Because two sequences (gallery and probe sequences) are required for recognition, dataset $\mathbf{A}$ is used hereafter.

${ }^{5}$ Since only a single gait feature was obtained for each dataset, statistical discriminant analysis considering within-class variance such as linear discriminant analysis could not be applied.
} 


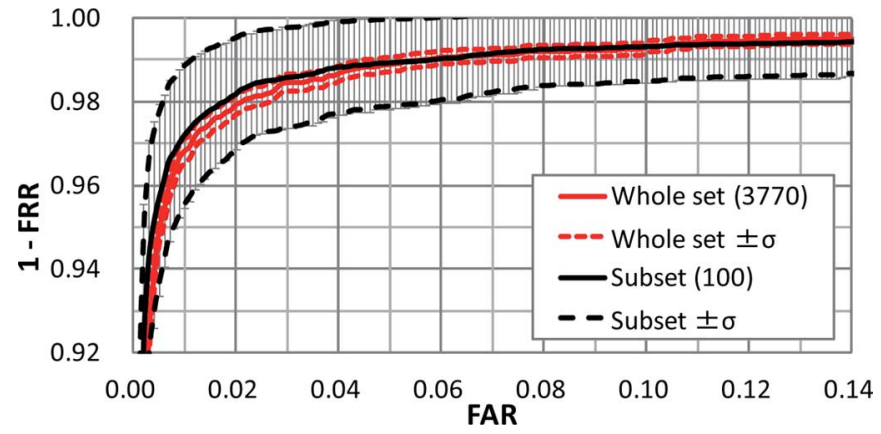

Fig. 10. ROC curves of GEI-based gait recognition with a varying number of subjects. Black and red indicate, respectively, smaller subsets and the whole set of A-65. The bold line and two bounding dashed lines indicate, respectively, the average $\mu$ and standard deviation range $\mu \pm \sigma$ derived from (4). Gray bars denote the standard deviation ranges $\mu \pm \sigma$ obtained in the experiments.

From statistical analysis of ROC curves [43], the standard deviation of the FRR with a single probe for each subject is estimated as

$$
\hat{\sigma}(\hat{p})=\sqrt{\frac{\hat{p}(1-\hat{p})}{n-1}},
$$

where $\hat{p}$ is the observed FRR and $n$ is the number of subjects. This indicates that the obtained FRR becomes more reliable as the number of subjects increases.

To validate the estimation, we repeated the experiments with randomly chosen subsets with fewer subjects and compared the actual standard deviation of the performance and that estimated from (4) using the GEI as the gait feature. First, we prepared 100 subsets comprising 100 subjects randomly chosen from dataset A-65 (which comprises 3,770 subjects) and obtained 100 ROC curves from the experimental results. We then calculated the average and standard deviation of the FRR for each FAR, depicted as an averaged ROC curve (bold black line) and standard deviation range bar (gray bar) in Fig. 10. Additionally, the estimated standard deviation range is depicted as two dashed black lines. From the graph, we see that the standard deviation ranges derived from the experimental results correspond well with those estimated from (4).

In addition, the results for the whole set are superimposed as the bold red line, while the standard deviation range estimated from (4) is depicted as two dashed red lines in Fig. 10. We see that the standard deviation range is significantly narrower than that of subsets with fewer subjects.

\section{B. Comparison of the Gait Feature}

1) Performance Comparison: This section compares the recognition performance of the six gait features described in Section IV-A. The recognition performance was evaluated using two metrics: (1) the ROC curve, and (2) the rank-1 and rank-5 identification rates. The rank- 1 and rank- 5 identification rates, which are common evaluation measures in a one-to-N matching scenario, denote the percentages of correct subjects out of all the subjects appearing within the first and fifth ranks, respectively. Note that the rank-1 and rank-5 identification rates depend on the gallery size, whereas the ROC curve is essentially independent of the gallery size.

First, the performance is compared for each observation angle using datasets A-55, A-65, A-75, and $\mathbf{A - 8 5}$, since the gait fea-
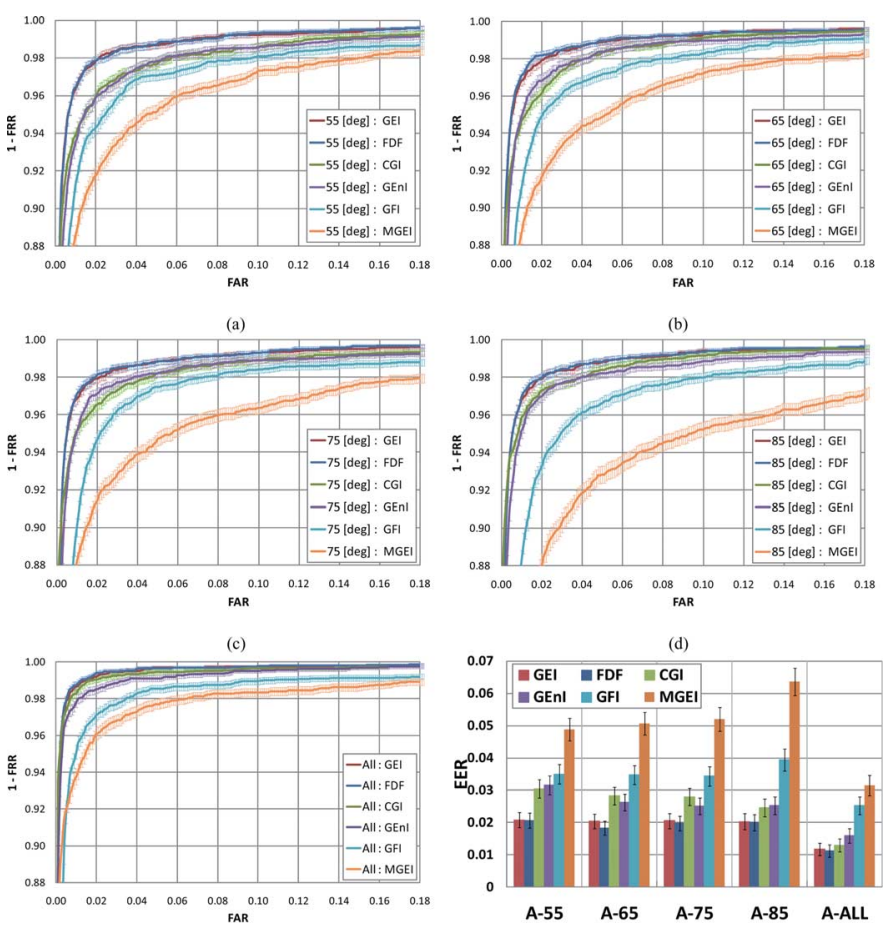

(e)

(f)

Fig. 11. Performance comparison of six gait features in terms of the ROC curve and EER. Each bar represents a standard deviation range derived from (4). (a) ROC for dataset A-55. (b) ROC for dataset A-65. (c) ROC for dataset A-75. (d) ROC for dataset A-85. (e) ROC for dataset A-ALL. (f) EERs for all the datasets.

ture property is dependent on the observation angle ${ }^{6}$. The ROC curves with standard deviation range bars for each dataset are shown in Fig. 11(a)-(d), while the Equal Error Rate (EER) is summarized in Fig. 11(f). In addition, rank-1 and rank-5 identification rates are given in Table IV. From the results, although the performances of the GEI and FDF are nearly equal and the performances of the GEnI and CGI are nearly equal, we see that there is a statistically significant performance difference between the GEI (or FDF), GEnI (or CGI), GFI, and MGEI, and the performance order of these techniques is almost independent of the observation angle.

Next, we compare the total performance using dataset A-ALL, with the results shown in Fig. 11(e) and Table IV (bottom row). As for the results for A-ALL, the following reasons are suggested for the improvement in recognition performance: a) the effect of gait fluctuations, which notably appears on the arm swing and head pose, was decreased by combining the scores of each observation angle, and b) the variations in the gait feature property caused by the observation angle improved the recognition performance, as reported in [44]. From these results, it can be seen that the GEI and FDF achieve the best performance overall.

Note that these comparison results are partly inconsistent with the results in previous works, for example, [6] (GEI versus CGI) and [8] (GEI versus GFI). The differences between the databases used for the evaluations (e.g., subject diversity, silhouette quality, sequence length, and intrasubject variations)

${ }^{6}$ For example, static features such as body shape are clearly seen in front-view gait images, while dynamic features such as the step and arm swing are clearly seen in side-view gait images. 
TABLE IV

Performance Comparison of Six Gait Features in Terms of the Rank-1 And Rank-5 Identification Rates

\begin{tabular}{lc|cccccc|cccccc}
\hline & & \multicolumn{4}{|c|}{ Rank-1 identification rate [\%] } & \multicolumn{5}{c}{ Rank-5 identification rate [\%] } \\
\cline { 3 - 13 } Dataset & \#Subjects & GEI & FDF & GEnI & CGI & GFI & MGEI & GEI & FDF & GEnI & CGI & GFI & MGEI \\
\hline \hline A-55 & 3,706 & $\mathbf{8 4 . 7 0}$ & 83.89 & 76.42 & 75.58 & 75.15 & 68.35 & $\mathbf{9 2 . 3 9}$ & 91.53 & 86.67 & 86.02 & 85.83 & 80.09 \\
A-65 & 3,770 & $\mathbf{8 6 . 6 3}$ & 85.49 & 78.65 & 78.97 & 77.11 & 68.91 & $\mathbf{9 2 . 8 4}$ & 92.81 & 88.14 & 88.06 & 87.32 & 79.71 \\
A-75 & 3,751 & $\mathbf{8 6 . 9 1}$ & 86.59 & 79.95 & 81.58 & 76.54 & 67.10 & 92.78 & $\mathbf{9 2 . 8 8}$ & 89.23 & 89.28 & 85.84 & 78.41 \\
A-85 & 3,249 & 85.72 & $\mathbf{8 5 . 9 0}$ & 80.95 & 83.35 & 74.92 & 61.19 & $\mathbf{9 3 . 0 1}$ & 92.83 & 89.60 & 90.80 & 84.73 & 73.19 \\
\hline A-ALL & 3,141 & $\mathbf{9 4 . 2 4}$ & 94.17 & 90.93 & 91.60 & 87.46 & 84.18 & $\mathbf{9 7 . 1 3}$ & 97.10 & 95.35 & 95.32 & 92.84 & 90.58 \\
\hline
\end{tabular}

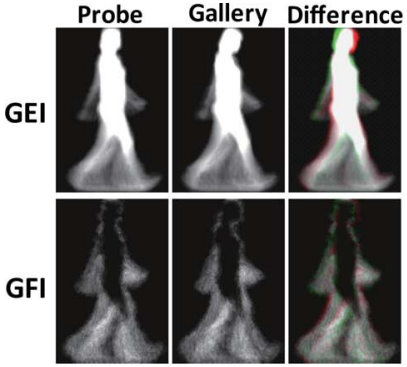

(a)

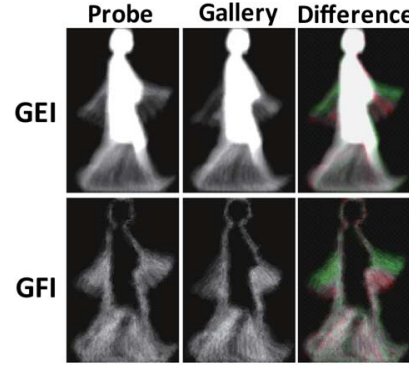

(b)
Fig. 12. Examples of subjects in A-85. Note that the value of the GFI is inverted for visibility. All feature differences between gallery and probe features are visualized by colors (green and red) in the corresponding difference image. Green indicates that the probe feature appears more strongly, while red depicts the opposite. Regarding the subject in (a), the rank score using the GEI is 216 , while that using the GFI is 1 (also, the rank scores are 79, 239, 7, and 19 using the FDF, CGI, GEnI, and MGEI, respectively). On the other hand, for the subject in (b), the rank score using the GEI is 1, while that using the GFI is 567 (also, the rank scores are all 1 using the other features). (a) A subject with large head pose fluctuation. (b) A subject with large arm swing fluctuation.

are considered to be the cause of the inconsistencies. For example, according to the latest evaluation results of the CGI reported in [45], GEI performance is superior to that of CGI only if there is no intrasubject variation and only a single gait period occurs in a sequence. Both these conditions are true in our dataset.

2) Correlation Among Features: Although some kind of upper limit on recognition performance using state-of-the-art gait features has been shown in the previous section, investigating the correlation among gait features is still meaningful for the design of a feature fusion scheme [46] to further improve recognition performance. Each gait feature has a unique property and is considered to be independent of other features to some extent. For example, Fig. 12(a) shows a subject in A-85 whose rank score is 216 using the GEI and 1 using the GFI. On the other hand, Fig. 12(b) shows a subject in A-85 whose rank score is 1 using the GEI and 567 using the GFI. These typical examples indicate that the GEI is relatively sensitive to static pose fluctuations and robust to motional fluctuation, while the GFI is the exact opposite.

To reveal which pair of features has a weak correlation, that is, is suitable for fusion, the rank score relations among gait features for each subject were analyzed. The results show that the GFI has relatively weak correlation with all the other features except the GEnI, and the CGI has the same with the GEnI, MGEI, and GFI. In addition, the GEnI has the same with the GEI, CGI, and MGEI. Some notable relations of rank scores for dataset A-85 among these features are shown in Fig. 13, while the relations of distances of the same subjects and different subjects are shown in Fig. 14. In the distance distributions shown in Fig. 14, though we can see that each distance relation between each pair of features is correlated as a whole, dispersal exists at a certain level. Therefore, these figures indicate that there is room for improvement in the recognition performance by fusing these gait features. Demonstration of this through fusion is one of our future works.

\section{Effects of Gender and Age}

This section investigates the difference in gait-recognition performance between genders and age groups. Our gait database is suited to this purpose because the age distribution of each gender is much wider than that in existing gait databases as mentioned in Section III-C. In this experiment, we adopted the GEI as the gait feature and carried out the evaluation on subset A-65, since it has the largest number of subjects in dataset $\mathbf{A}^{7}$.

Ages were grouped in 5-year intervals up to 20 years of age and in 10-year intervals from 20 to 60 years of age for each gender $^{8}$. Ages over 60 years were treated as one age group because of the shortage of subjects. The numbers of subjects in each gender/age group are given in Table $\mathrm{V}$.

The EER for each gender/age group is depicted in Fig. 15, while the distance distributions of the same subjects (true attempts) for each group are shown in Fig. 16. A comparison of the distance distributions of the same subjects and different subjects (imposters) for four typical age groups - under 10s (5 to 9 years old), early 10s, 20s, and 40s-are depicted in Fig. 17. Note that the original L2 norm (3) is shown in these distributions.

1) Effect of Gender: First, we focus on the difference in gait-recognition performance between males and females. According to the results in Fig. 15, recognition performance for females tends to be better than that for males in almost all the age groups. Additionally, Fig. 17 implies that the intersubject variation in females' gait is greater than that in males' gait, while intrasubject variations are not that different between males and females in each age group. The difference in intrasubject variation is assumed to be due to the fact that the range of appearance variation in females, which mainly comes from variations in hair style, clothes, and shoes, is greater than that in males.

\footnotetext{
${ }^{7}$ We also carried out this experiment using all the other gait features described in Section IV-A on another subset $\mathbf{A - 8 5}$, but the results showed similar trends.

${ }^{8}$ Taking the rapid physical growth rate into consideration, we used 5 year intervals up to 20 years to reveal more detailed changes in recognition performance during the growing process.
} 


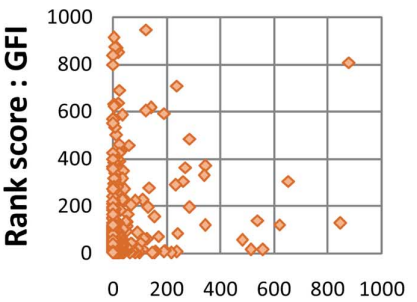

Rank score: GEI

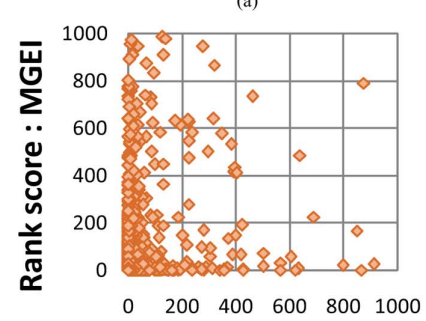

Rank score: GFI

(c)

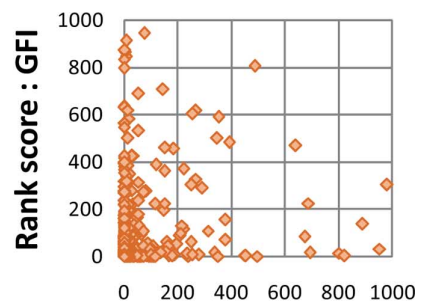

Rank score: $\mathbf{C G I}$

(b)

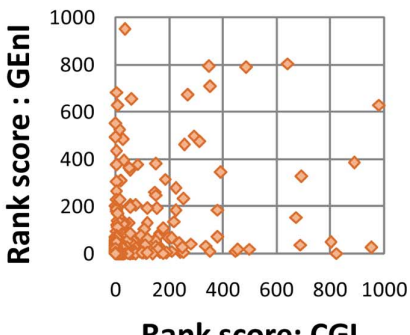

(d)

Fig. 13. Examples of the rank score correlations between pairs of gait features. (a) GEI and GFI. (b) CGI and GFI. (c) GFI and MGEI. (d) CGI and GEnI.

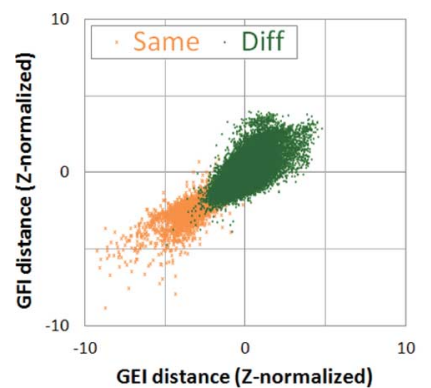

(a)

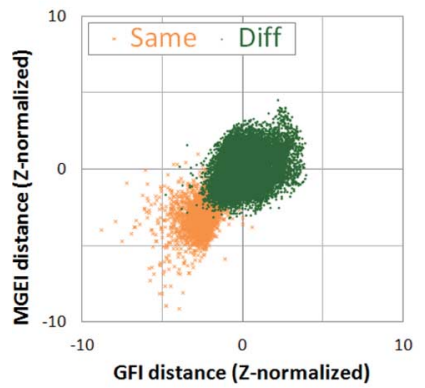

(c)

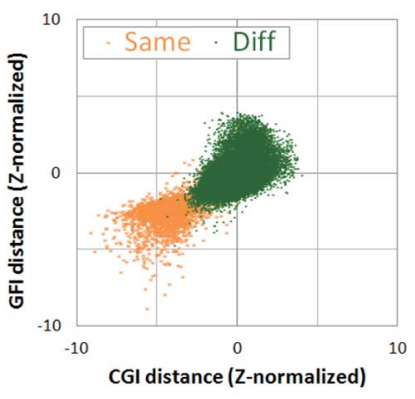

(b)

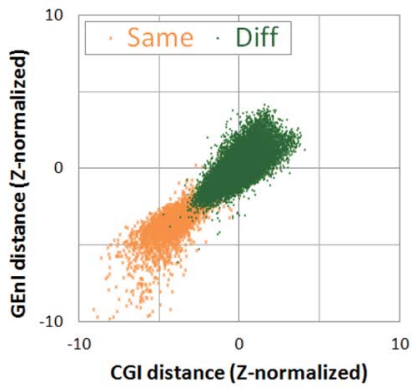

(d)
Fig. 14. Examples of the distance correlations between pairs of gait features. (a) GEI and GFI. (b) CGI and GFI. (c) GFI and MGEI. (d) CGI and GEnI.

2) Effect of Age: Next, we focus on the difference in gaitrecognition performance between age groups. From the results in Fig. 15, we see that gait-recognition performance for the group of very young children ( 0 to 4 years old) is worse than that for the other age groups, and this gradually improves with the slightly older groups up to the group of late $10 \mathrm{~s}$. This result is intuitively understandable because the intrasubject gait fluctuation for children is greater owing to the immaturity of their walking, as illustrated in Fig. 16. On the other hand, the fluctuation in gait for adults is small as shown in Fig. 16. This indicates that adults have established their own walking style; in other words, they have a fixed gait pattern. In this regard, however, the intrasubject variation in the over-60 female group is slightly larger than that in other adult age groups, and this is assumed

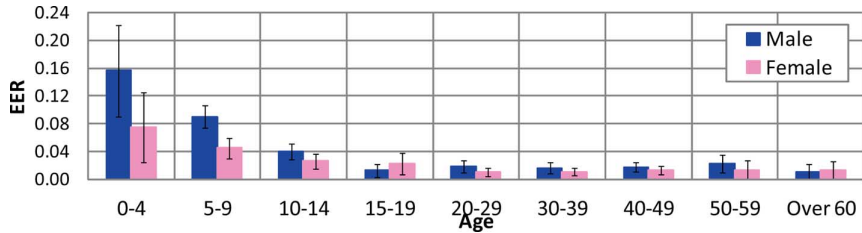

Fig. 15. EERs among genders and age groups. The black bars represent the standard deviation ranges derived from (4).
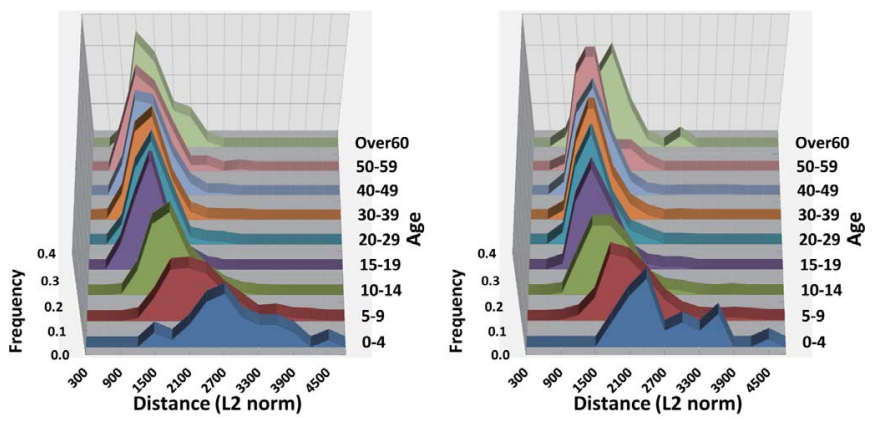

Fig. 16. Distance distributions of the same subjects in each gender/age group. (a) Male. (b) Female.
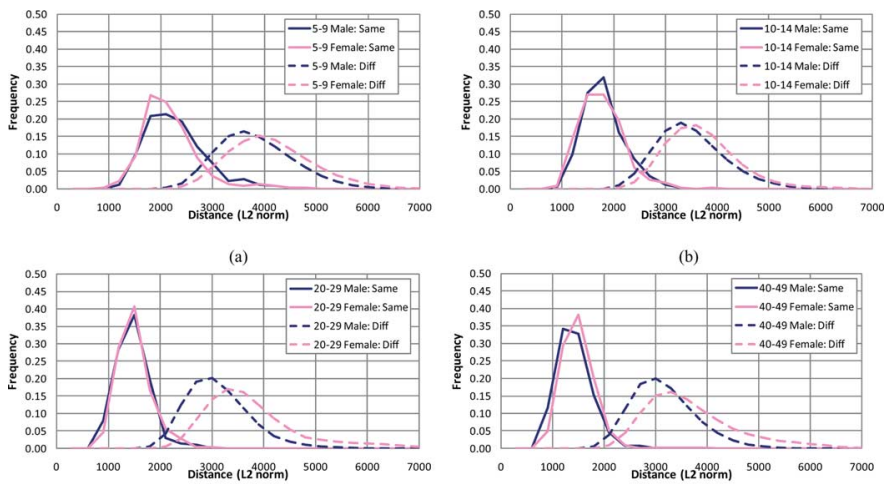

(c)

Fig. 17. Comparison of distance distributions of the same subjects and different subjects in four typical age groups. (a) Group of 5 to 9 year olds. (b) Group of early 10 s. (c) Group of 20 s. (d) Group of 40 s.

to be due to a decline in physical strength with aging. Further study of elderly groups (over 60 years old), together with the additional data collection required, is considered as future work. In addition, a more detailed analysis of gait properties among age groups, such as investigation of the differences in the effects of body parts among age groups, is one of our future works.

The above observations indicate that the dependence of gait fluctuation on the age group implies that gait fluctuation can be a useful cue for age classification according to gait. In addition, the age group can be regarded as a so-called quality measure [47] for gait recognition, which is one of the interesting future directions of this study.

\section{CONCLUSION}

This paper described the construction of a gait database comprising a large population dataset and presented a statistically reliable performance evaluation of vision-based gait recognition. This dataset has the following advantages over existing gait databases: (1) the number of subjects is 4,007 , which is more than 20 times greater than the number in existing public largescale databases, (2) the male-to-female ratio is close to $1,(3)$ the 
TABLE V

Numbers of SubJects of EAch Gender and Age Group in A-65

\begin{tabular}{l|ccccccccc}
\hline & \multicolumn{8}{|c}{ Age } \\
Gender & $0-4$ & $5-9$ & $10-14$ & $15-19$ & $20-29$ & $30-39$ & $40-49$ & $50-59$ & Over 60 \\
\hline \hline Male & 32 & 312 & 323 & 150 & 267 & 288 & 412 & 134 & 89 \\
Female & 29 & 213 & 226 & 90 & 285 & 369 & 403 & 73 & 75 \\
\hline
\end{tabular}

age distribution is wide, ranging from 1 to 94 years, and (4) the quality of all silhouettes is guaranteed by visual confirmation. Using our dataset, we carried out a statistically reliable performance comparison of gait recognition using state-of-the-art gait features. Moreover, the dependence of recognition performance on gender and age group was analyzed with the results providing several new insights, including the performance difference between males and females, and the gradual change in recognition performance with human growth.

Although our dataset has the largest population of all databases till now, there is still an insufficient number of very young children and elderly persons when compared with the numbers of other generations. Therefore, we need to collect the required gait datasets by taking advantage of various events, such as outreach activities, in the future. Additionally, the construction of another dataset using images taken with camera 2 is a future work.

Moreover, further analysis of gait recognition performance using our dataset is still needed. For example, our dataset enables the evaluation of cross-view recognition and this will show the robustness of each gait feature with respect to view variations. Finally, our database is suitable for the development of gait-based gender and age classification algorithms, which are quite meaningful for many vision applications such as intelligent surveillance, and these remain as future works.

\section{REFERENCES}

[1] G. Zhao, G. Liu, H. Li, and M. Pietikainen, "3D gait recognition using multiple cameras," in Proc. 7th Int. Conf. Automatic Face and Gesture Recognition, Apr. 2006, pp. 529-534.

[2] G. Veres, L. Gordon, J. Carter, and M. Nixon, "What image information is important in silhouette-based gait recognition?," in Proc. IEEE Conf. Computer Vision and Pattern Recognition, 2004, vol. 2, pp. 776-782.

[3] Z. Liu and S. Sarkar, "Improved gait recognition by gait dynamics normalization," IEEE Trans. Pattern Anal. Mach. Intell., vol. 28, no. 6, pp. 863-876, Jun. 2006.

[4] Y. Makihara, R. Sagawa, Y. Mukaigawa, T. Echigo, and Y. Yagi, "Gait recognition using a view transformation model in the frequency domain," in Proc. 9th Eur. Conf. Computer Vision, Graz, Austria, May 2006, pp. 151-163.

[5] K. Bashir, T. Xiang, and S. Gong, "Gait recognition using gait entropy image," in Proc. 3rd Int. Conf. Imaging for Crime Detection and Prevention, 2009.

[6] C. Wang, J. Zhang, J. Pu, X. Yuan, and L. Wang, "Chrono-gait image: A novel temporal template for gait recognition," in Proc. 11th Eur. Conf. Computer Vision, 2010, pp. 257-270.

[7] K. Bashir, T. Xiang, and S. Gong, "Gait recognition without subject cooperation," Pattern Recognit. Lett., vol. 31, no. 13, pp. 2052-2060, 2010.

[8] T. H. W. Lam, K. H. Cheung, and J. N. K. Liu, "Gait flow image: A silhouette-based gait representation for human identification," Pattern Recognit., vol. 44, no. 4, pp. 973-987, 2011.

[9] J. Wang, M. She, S. Nahavandi, and A. Kouzani, "A review of visionbased gait recognition methods for human identification," in Proc. Int. Conf. Digital Image Computing: Techniques and Applications, 2010, pp. $320-327$.
[10] S. Yu, T. Tan, K. Huang, K. Jia, and X. Wu, "A study on gait-based gender classification," IEEE Trans. Image Process., vol. 18, no. 8, pp. 1905-1910, Aug. 2009.

[11] J. Lu and Y.-P. Tan, "Gait-based human age estimation," IEEE Trans. Inf. Forensics Security, vol. 5, no. 4, pp. 761-770, Oct. 2010.

[12] R. Gross and J. Shi, The CMU Motion of Body (Mobo) Database CMT, Tech. Rep., 2001.

[13] T. Chalidabhongse, V. Kruger, and R. Chellappa, The umd Database for Human Identification at a Distance University of Maryland, Tech. Rep., 2001.

[14] J. Shutler, M. Grant, M. Nixon, and J. Carter, "On a large sequencebased human gait database," in Proc. Fourth Int. Conf. Recent Advances in Soft Computing, 2002, pp. 66-72.

[15] J. B. Hayfron-Acquah, M. S. Nixon, and J. N. Carter, "Automatic gait recognition by symmetry analysis," Pattern Recognit. Lett., vol. 24, pp. 2175-2183, 2003.

[16] L. Wang, T. Tan, H. Ning, and W. Hu, "Silhouette analysis-based gait recognition for human identification," IEEE Trans. Pattern Anal. Mach. Intell., vol. 25, no. 12, pp. 1505-1518, Dec. 2003.

[17] R. Tanawongsuwan and A. Bobick, A Study of Human Gaits Across Different Speeds Georgia Tech, Tech. Rep., 2003.

[18] S. Sarkar, J. Phillips, Z. Liu, I. Vega, P. Grother, and K. Bowyer, "The humanid gait challenge problem: Data sets, performance, and analysis," IEEE Trans. Pattern Anal. Mach. Intell., vol. 27, no. 2, pp. 162-177, Feb. 2005.

[19] S. Yu, D. Tan, and T. Tan, "A framework for evaluating the effect of view angle, clothing and carrying condition on gait recognition," in Proc. 18th Int. Conf. Pattern Recognition, Hong Kong, China, Aug. 2006, vol. 4, pp. 441-444.

[20] D. Tan, K. Huang, S. Yu, and T. Tan, "Efficient night gait recognition based on template matching," in Proc. 18th Int. Conf. Pattern Recognition, 2006, vol. 3, pp. 1000-1003.

[21] D. Matovski, M. Nixon, S. Mahmoodi, and J. Carter, "The effect of time on the performance of gait biometrics," in Proc. IEEE Fourth Conf. Biometrics: Theory, Applications and Systems, Washington, DC, Sep. 2010, pp. 1-6.

[22] Y. Makihara, A. Tsuji, and Y. Yagi, "Silhouette transformation based on walking speed for gait identification," in Proc. 23rd IEEE Conf. Computer Vision and Pattern Recognition, San Francisco, CA, Jun. 2010.

[23] M. A. Hossain, Y. Makihara, J. Wang, and Y. Yagi, "Clothing-invariant gait identification using part-based clothing categorization and adaptive weight control," Pattern Recognit., vol. 43, no. 6, pp. 2281-2291, Jun. 2010.

[24] A. Mori, Y. Makihara, and Y. Yagi, "Gait recognition using period-based phase synchronization for low frame-rate videos," in Proc. 20th Int. Conf. Pattern Recognition, Istanbul, Turkey, Aug. 2010, pp. 2194-2197.

[25] H. Mannami, Y. Makihara, and Y. Yagi, "Gait analysis of gender and age using a large-scale multi-view gait database," in Proc. 10th Asian Conf. Computer Vision, Queenstown, New Zealand, Nov. 2010.

[26] M. Okumura, H. Iwama, Y. Makihara, and Y. Yagi, "Performance evaluation of vision-based gait recognition using a very large-scale gait database," in Proc. IEEE Fourth Int. Conf. Biometrics: Theory, Applications and Systems, 2010.

[27] Y. Makihara, M. Okumura, H. Iwama, and Y. Yagi, "Gait-based age estimation using a whole-generation gait database," in Proc. Int. Joint Conf. Biometrics, 2011, pp. 1-6.

[28] D. Matovski, M. Nixon, S. Mahmoodi, and J. Carter, "The effect of time on gait recognition performance," IEEE Trans. Inf. Forensics Security, vol. 7, no. 2, pp. 543-552, Apr. 2011.

[29] I. Bouchrika and M. Nixon, "Exploratory factor analysis of gait recognition," in Proc. 8th IEEE Int. Conf. Automatic Face and Gesture Recognition, Amsterdam, The Netherlands, 2008.

[30] S. Yu, D. Tan, and T. Tan, "Modelling the effect of view angle variation on appearance-based gait recognition," in Proc. 7th Asian Conf. Computer Vision, Jan. 2006, vol. 1, pp. 807-816. 
[31] M. Okumura, Y. Makihara, S. Nakamura, S. Morishima, and Y. Yagi, "The online gait measurement for the audience-participant digital entertainment," in Invited Workshop on Vision Based Human Modeling and Synthesis in Motion and Expression, Xi' an, China, Sep. 2009.

[32] S. Morishima, Y. Yagi, and S. Nakamura, "Instant movie casting with personality: Dive into the movie system," in Proc. 2011 Int. Conf. Virtual and Mixed Reality: Systems and Applications, 2011, pp. 187-196.

[33] Z. Liu, L. Malave, and S. Sarkar, "Studies on silhouette quality and gait recognition," in Proc. IEEE Conf. Computer Vision and Pattern Recognition, 2004, vol. 2, pp. 704-711.

[34] Z. Liu and S. Sarkar, "Effect of silhouette quality on hard problems in gait recognition," IEEE Trans. Syst., Man, Cybern. B, Cybern., vol. 2, no. 2, pp. 170-183, Apr. 2004.

[35] Y. Boykov and M. Jolly, "Interactive graph cuts for optimal boundary and region segmentation of objects in n-d images," in Proc. Int. Conf. Computer Vision, 2001, pp. 105-112.

[36] Z. Zhang, "A flexible new technique for camera calibration," IEEE Trans. Pattern Anal. Mach. Intell., vol. 22, no. 11, pp. 1330-1334, Nov. 2000.

[37] S. Tsuji, Y. Yagi, and M. Asada, "Dynamic scene analysis for a mobile robot in a man-made environment," in Proc. IEEE Int. Conf. Robotics and Automation, 1985 , pp. $850-855$.

[38] M. Magee and J. Aggarwal, "Determining vanishing points from perspective images," Comput. Vis., Graph., Image Process., vol. 26, no. 2, pp. 256-267, 1984.

[39] Z. Liu and S. Sarkar, "Simplest representation yet for gait recognition: Averaged silhouette," in Proc. 17th Int. Conf. Pattern Recognition, Aug. 2004, vol. 1, pp. 211-214.

[40] J. Han and B. Bhanu, "Individual recognition using gait energy image," IEEE Trans. Pattern Anal. Mach. Intell., vol. 28, no. 2, pp. 316-322, Feb. 2006.

[41] P. Grother, Face Recognition Vendor Test 2002 Supplemental Report 2004 [Online]. Available: http://www.face-rec.org/vendors

[42] J. Kittler, M. Hatef, R. P. W. Duin, and J. Matas, "On combining classifiers," IEEE Trans. Pattern Anal. Mach. Intell., vol. 20, no. 3, pp. 226-239, Mar. 1998.

[43] G. Snedecor and W. Cochran, Statitical Methods. Ames, IA: Iowa State Univ. Press, 1967.

[44] K. Sugiura, Y. Makihara, and Y. Yagi, "Gait identification based on multi-view observations using omnidirectional camera," in Proc. 8th Asian Conf. Computer Vision, Nov. 2007, pp. 452-461.

[45] C. Wang, J. Zhang, L. Wang, J. Pu, and X. Yuan, "Human identification using temporal information preserving gait template," IEEE Trans. Pattern Anal. Mach. Intell., 2011, DOI: 10.1109/TPAMI.2011.260.

[46] A. A. Ross, K. Nandakumar, and A. K. Jain, Handbook of Multibiometrics (International Series on Biometrics). New York: SpringerVerlag, 2006.

[47] P. Grother and E. Tabassi, "Performance of biometric quality measures," IEEE Trans. Pattern Anal. Mach. Intell., vol. 29, no. 4, pp. 531-543, Apr. 2007.

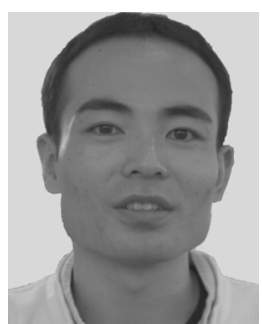

Haruyuki Iwama received the B.S. and M.S. degrees in engineering science from Osaka University in 2003 and 2005, respectively.

In 2005, he joined the Production Technology Development Group, Sharp Corporation, where he worked on vision-based inspection systems. $\mathrm{He}$ is currently a Ph.D. candidate and a Specially Appointed Researcher of the Institute of Scientific and Industrial Research, Osaka University. His research interests are multiview shadow and foreground segmentation, group context-based person identification, and gait recognition.

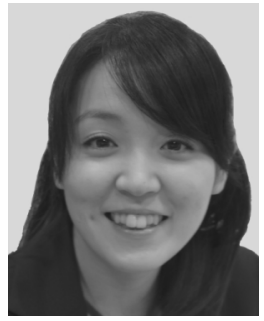

Mayu Okumura received the B.S. and M.S. degrees in information science from Osaka University in 2009 and 2011, respectively.

Her research interests are gait personality measurement for digital entertainment and performance evaluation of gait recognition and gait-based age estimation with the large population database.

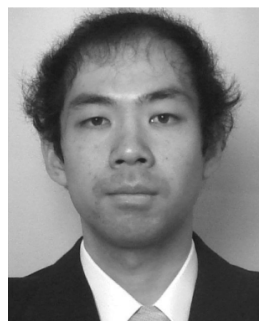

Yasushi Makihara received the B.S., M.S., and $\mathrm{Ph} . \mathrm{D}$. degrees in engineering from Osaka University in 2001, 2002, and 2005, respectively.

$\mathrm{He}$ is currently an Assistant Professor of the Institute of Scientific and Industrial Research, Osaka University. His research interests are gait recognition, morphing, and temporal super resolution. He is a member of IPSJ, RJS, and JSME.

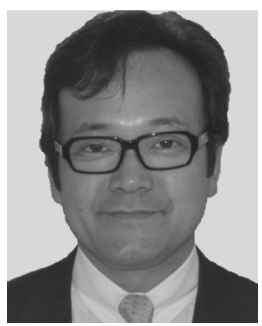

Yasushi Yagi (M'91) received the Ph.D. degree from Osaka University in 1991.

$\mathrm{He}$ is the Director of the Institute of Scientific and Industrial Research, Osaka university, Ibaraki, Japan. In 1985, he joined the Product Development Laboratory, Mitsubishi Electric Corporation, where he worked on robotics and inspections. He became a Research Associate in 1990, a Lecturer in 1993, an Associate Professor in 1996, and a Professor in 2003 at Osaka University. International conferences for which he has served as Chair include: FG1998 (Financial Chair), OMINVIS2003 (Organizing chair), ROBIO2006 (Program cochair), ACCV2007 (Program chair), PSVIT2009 (Financial chair), ICRA2009 (Technical Visit Chair), ACCV2009 (General chair), ACPR2011 (Program cochair), and ACPR2013 (General chair). He has also served as the Editor of IEEE ICRA Conference Editorial Board (2007-2011). He is the Editorial member of IJCV and the Editor-in-Chief of IPSJ Transactions on Computer Vision \& Applications. His research interests are computer vision, medical engineering, and robotics.

Dr. Yagi was awarded the ACM VRST2003 Honorable Mention Award, IEEE ROBIO2006 Finalist of T.J. Tan Best Paper in Robotics, IEEE ICRA2008 Finalist for Best Vision Paper, MIRU2008 Nagao Award, and the PSIVT2010 Best Paper Award. He is a fellow of IPSJ and a member of IEICE and RSJ. 